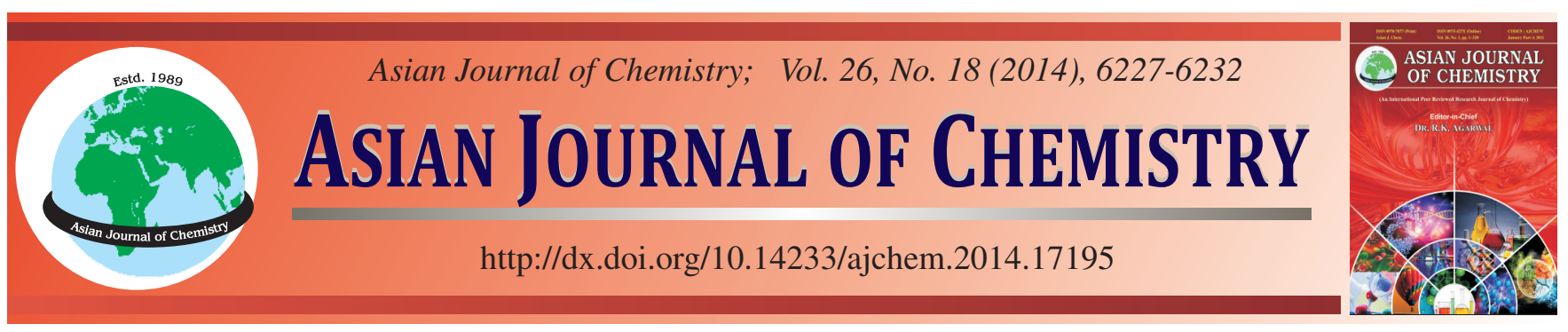

\title{
Molecular Docking Studies and Comparative Binding Mode Analysis of FDA Approved HIV Protease Inhibitors
}

Pran Kishore Deb ${ }^{*}$, Ahmad Junaid, Dina El-Rabie, Tan Yee Hon, Elham Mohammadi Nasr and Mallikarjuna Rao Pichika

Department of Pharmaceutical Chemistry, School of Pharmacy, International Medical University, 126, Jalan Jalil Perkasa 19, Bukit Jalil, Kuala Lumpur 57000, Malaysia

*Corresponding author: E-mail: prankishore@imu.edu.my; prankishore1@gmail.com

\begin{abstract}
The HIV protease enzyme (maturation enzyme) is one of the most promising therapeutic targets for the treatment of AIDS. Due to the mutation in the virus, there is always room for new agents. In the present study, in silico molecular docking study was carried to analyze and compare the binding mode of nine FDA approved HIV protease inhibitors as well as to understand their structural requirements to inhibit the enzyme by employing the Glide module of Schrodinger software. Prime MM-GBSA approach was used to study the free energy of binding of these inhibitors with the enzyme. QikProp module of Schrodinger was used to predict the ADME properties of study compounds to evaluate their drug likeness. Binding mode analysis shows that the active site is present at the interface of two chains (A and B) of the enzyme and the crucial amino acid residues responsible for the binding of inhibitors to the HIV-1 protease are found to be Asp25, Gly27, Ala28, Asp29 and Gly49, respectively. Also the ADME properties predicted was found to be in ranges predicted by QikProp module of Schrodinger software for $95 \%$ of the known oral drugs. Results of this comparative binding mode analysis of all the FDA approved drugs could be potential and useful for the design of new potent inhibitors of HIV-1 protease.
\end{abstract}

Keywords: FDA approved HIV protease inhibitors, Docking studies, in silico ADME prediction.

ᄂ - - - - - - - - - - - - - - - - - - - - - - - - - - - - -

\section{INTRODUCTION}

It is well established that acquired immuno deficiency syndrome (AIDS) is one of the most fatal transmissible disease ${ }^{1}$ caused by human immunodeficiency virus Type-1 (HIV-1) which is a slow progressing retrovirus (lentivirus) ${ }^{2}$. To fight with this deadly disease there are several groups of anti-HIV drugs approved by Food and Drug Administration (FDA) which slows the progression of disease and even in some cases mortality of HIV infection ${ }^{3}$. These are protease inhibitors (PI's), fusion inhibitors (FI's), co-receptor inhibitors (CRI's), integrase inhibitors (INI's), nucleoside reverse transcriptase inhibitors (NRTI's), nucleotide reverse transcriptase inhibitors and nonreverse transcriptase inhibitors ${ }^{4}$. These groups of drugs are based on the basic enzymes of the HIV life cycle. Currently the most favourable anti-HIV therapy is the combination of these inhibitors called HAART (highly active anti-retroviral therapy) $)^{3}$. One of the most important life cycle enzymes of the HIV is HIV-1 protease. It is retropepsin enzyme which helps the infectious HIV-virion by maturing the protein components. HIV virus remains un-infectious without operative HIV protease $^{5}$. Thus protease inhibitors inhibit the maturation of the progeny virions. Structurally, HIV protease is a protein containing two similar chains A and B (homodimer) ${ }^{4}$ and each chain containing 99 amino acids ${ }^{3}$. The active site of this homodimer contains aspartic acid (Asp) residue in both the chains which shows that the active site is present at the dimer interface, so, catalytically it acts as an aspartic acid protease ${ }^{4}$. The crucial amino acids responsible for the binding of inhibitors to the protein are Asp25, Gly27, Ala28, Asp29 and Gly49³, respectively. Currently, there are 10 FDA approved protease inhibitors i.e., Amprenavir ${ }^{6}$, darunavir ${ }^{7}$, indinavir ${ }^{8}$, nelfinavir $^{9}$, ritonavir $^{10}$, atazanavir ${ }^{11}$ tipranavir $^{12}$, lopinavir/ritonavir ${ }^{13}$, saquinavir $^{14}$ and fosamprenavir ${ }^{15}$, respectively (Table-1). All the protease inhibitors except tipranavirare peptidomimetic drugs based on non-hydrolyzable transition state isostere ${ }^{4}$. In the present study, in silico molecular docking studies (using the crystal structure of enzyme HIV-protease, PDB ID: 3 EKV), comparative binding mode analysis and ADME predictions of all the FDA approved protease inhibitors (Table-1) have been carried out. This study provides an insight into the physiochemical and structural requirements of inhibitors for effective binding to the enzyme protease which could be helpful for the design and development of new potent protease inhibitors to overcome the problem of resistance and cross-resistance ${ }^{4}$ to present drugs. 
TABLE-1

FREE ENERGY OF BINDING OF THE COMPOUNDS (INHIBITORS) WITH THE ENZYME HIV-1 PROTEASE (PDB ID: 3EKV)

\begin{tabular}{|c|c|c|c|c|c|c|c|}
\hline Compound & $\mathrm{IC}_{50}(\mu \mathrm{M})$ & $\begin{array}{c}\text { Prime } \\
\text { MMGBSA } \\
\text { complex } \\
\text { energy }\end{array}$ & $\begin{array}{c}\text { Prime } \\
\text { MMGBSA } \\
\text { ligand energy }\end{array}$ & $\begin{array}{l}\text { Prime MMGBSA } \\
\text { receptor energy }\end{array}$ & $\begin{array}{c}\text { Prime MMGBSA } \\
\text { DG bind } \\
\left(\Delta \mathrm{G}_{\text {bind }}=\right. \\
\mathrm{kcal} / \mathrm{mol})\end{array}$ & $\begin{array}{c}\text { Prime } \\
\text { MMGBSA } \\
\text { DG bind } \\
\text { coulomb }\end{array}$ & $\begin{array}{c}\text { Prime } \\
\text { MMGBSA } \\
\text { DG bind } \\
\text { vdW }\end{array}$ \\
\hline Amprenavir & $0.021 \pm 0.007$ & -8275.92 & -45.2705 & -8112.61 & -118.042 & -8.05039 & -72.5866 \\
\hline Darunavir & $1.2 \pm 0.8$ & -8242.15 & -28.3099 & -8112.61 & -101.226 & -16.0765 & -56.2796 \\
\hline
\end{tabular}

\begin{tabular}{llllllll} 
& \\
\hline
\end{tabular}

HN

络

$Y^{\prime \prime \prime}=0$

(1)

Atazanavir

$0.84 \pm 0.16 \quad-8307.48$

$-141.401$

$-8112.61$

$-53.4677$

$-29.6322$

$-43.948$
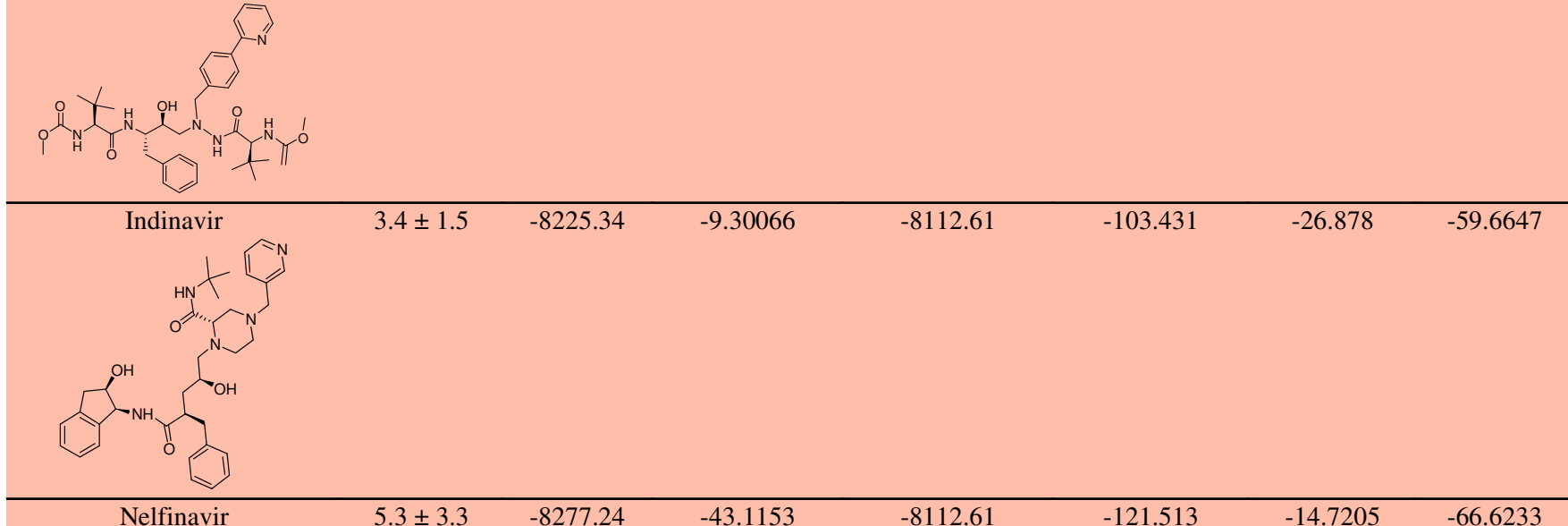

\begin{tabular}{|c|c|c|c|c|c|c|c|}
\hline Nelfinavir & $5.3 \pm 3.3$ & -8277.24 & -43.1153 & -8112.61 & -121.513 & -14.7205 & -66.6233 \\
\hline Ritonavir & $4.6 \pm 1.4$ & -8357.42 & -140.417 & -8112.61 & -104.391 & -31.2891 & -73.8022 \\
\hline Saquinavir & $1.0 \pm 0.5$ & -8344.78 & -123.116 & -8112.61 & -109.055 & -27.1336 & -60.9611 \\
\hline Tipranavir & $3.2 \pm 1.2$ & -8269.22 & -55.8455 & -8112.61 & -100.759 & -1.751511 & -59.1267 \\
\hline
\end{tabular}




\section{EXPERIMENTAL}

Molecular modelling study: Molecular modeling investigations were carried out using Dell Precision work station T3400 running Intel Core2 Duo Processor, 4GB RAM, 250 GB hard disk and NVidia Quodro FX 4500 graphics card. Maestro 9.4, GLIDE v5.9 XP docking program (Schrodinger Inc.) was employed for the docking studies ${ }^{16-18}$.

Preparation of protein: Crystal structure of HIV-1 protease (PDB ID: $3 \mathrm{EKV})^{3}$ was downloaded from the protein data bank (PDB) (www.rcsb.org), refined and prepared using Schrodinger protein preparation wizard tool (Glide v5.9), which performs the following steps: Assigning of bond orders, addition of hydrogens, optimization of hydrogen bonds by flipping amino side chains, correction of charges and minimization of the protein complex. All the bound water molecules, ligands and co-factors were removed (preprocess) from the proteins which were taken in .mae format. The tool neutralized the side chains that are not close to the binding cavity and do not participate in salt bridges. This step is then followed by restrained minimization of co-crystallized complex, which reorients side chain hydroxyl groups and alleviates potential steric clashes. The complex obtained was minimized using OPLS_2005 force field23 with Polack-Ribiere Conjugate Gradient (PRCG) algorithm. The minimization was terminated either completion of 5,000 steps (or) after the energy gradient converged below $0.05 \mathrm{kcal} / \mathrm{mol}$.

Preparation of ligands: Structures of the ligands (Table-1) were sketched using built panel of Maestro and taken in .mae format. LigPrep is a utility of Schrodinger software suit that combines tools for generating 3D structures from 1D (Smiles) and 2D (SDF) representation, searching for tautomers, steric isomers and perform a geometry minimization of the ligands. Molecular Mechanics Force Fields (OPLS_2005) with default settings were employed for the ligand minimization.

Docking studies: Docking studies were carried out by using the above mentioned prepared proteins (PDB ID: 3 EKV) and ligands (Table-1), by employing Glide XP docking program (Schrodinger Inc.) following the reported procedure ${ }^{16}$.

Calculation of Prime MM-GBSA descriptors: The Prime MM-GBSA approach ${ }^{19}$ is used to predict the free energy of binding for a receptor and a set of ligands. MM-GBSA is an acronym for a method that combines OPLS molecular mechanics energies (EMM), an SGB solvation model for polar solvation (GSGB) and a nonpolar solvation term (GNP) composed of the nonpolar solvent accessible surface area and van der Waals interactions. The total free energy of binding is then expressed as:

$$
\Delta \mathrm{G}_{\text {bind }}=\mathrm{G}_{\text {complex }}-\left(\mathrm{G}_{\text {protein }}+\mathrm{G}_{\text {ligand }}\right)
$$

where

$$
\mathrm{G}=\mathrm{EMM}+\mathrm{GSGB}+\mathrm{GNP}
$$

The ligand in the unbound state is minimized in SGB solvent but is not otherwise sampled. In the calculation of the complex, the ligand is minimized in the context of the receptor. The protein is currently held fixed in all calculations. The following descriptors generated by the Prime MM-GBSA approach:

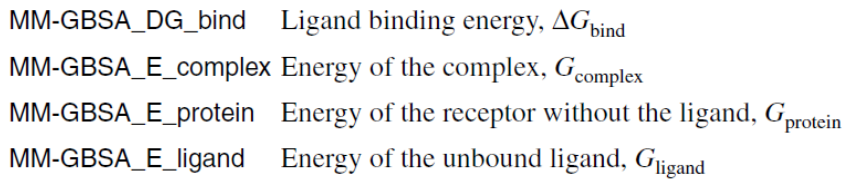

To set up the calculation, pose viewer file (generated after docking with Glide) was used to consider the receptor and source of ligands and the program Prime MM-GBSA was run with default options that were chosen to produce reasonable descriptors. The MM-GB/SA scoring along with the experimental binding affinities data of HIV-protease is presented in Table-1.

Prediction of ADME properties: The QikProp module of Schrodinger is a quick, accurate, easy-to-use absorption, distribution, metabolism and excretion (ADME) prediction program design to produce certain descriptors related to ADME. QikProp predicts physically significant descriptors and pharmaceutically relevant properties of organic molecules, either individually or in batches. In the present study, QikProp was run in normal processing mode with default options ${ }^{20}$. The selected properties that are known to influence metabolism, cell permeation and bioavailability are presented in Table-3.

\section{RESULTS AND DISCUSSION}

In the present work, a structure based molecular docking studies have been carried out for analyzing and comparing the binding mode of various FDA approved inhibitors of the essential enzyme HIV-1 protease to gain insight into the physiochemical and structural requirements for effective binding with the enzyme which can be useful in identifying new potent inhibitors. The docking protocol has been validated before carrying out docking studies by reproducing the natural bound substrate conformation amprenavir in the crystal structure of HIV-1 protease (PDB ID: 3 EKV). The co-crystal (amprenavir) was taken out of the active site and re-docked. The top 3 docking configurations were taken into account to validate the results and RMSD was calculated for each configuration in comparison with the co-crystallized ligand. The acceptable results (RMSD within 1.12-1.88 ) indicated the docking configurations have similar binding positions and orientations within the binding site and are similar to the co-crystal structure, which illustrate the fact that the docking protocols used could be successfully generated the co-crystal-HIV-1 protease complex precisely.

To study the association and the free energy of the binding of these ligands to the enzyme, Prime MM-GBSA method was used (Table-1). Moreover, ADME descriptors were also used to get better insight into the physio-chemical requirements for effective binding of ligands to HIV-1 protease (Table-3).

The binding interactions of all the inhibitors are explained by highlighting their hydrogen bonding interactions with the enzyme protease. The binding orientation of the co-crystal ligand (Fig. 1a) shows that the amprenavir forms four essential hydrogen bonding interactions inside the active site of chain A of the protease enzyme. The carbonyl group has shown hydrogen bonding interaction with the amino acid residue Gly49 at a distance of $2.593 \AA$. At the same time, the hydroxyl 
group and oxygen atom of tetrahydrofuran ring formed hydrogen bond with the amino acid residues Asp25 and Asp29 at a distance of $1.716 \AA$ and $2.068 \AA$, respectively. The amine group was also found to form a hydrogen bond with Ala28 at a distance of $3.242 \AA$ (Fig. 1a). Similarly, in case of darunavir (Fig. 1b) the carbonyl group and amino group $\left(\mathrm{NH}_{2}\right.$ of parasulfonamido moiety) was found to form significant hydrogen bonding interaction with the amino acid residues Gly49 (2.155 $\AA$ ) and Asp30 (2.088 ̊), respectively. Interestingly, the free hydroxyl group $(-\mathrm{OH})$ forms two very crucial hydrogen bonding interactions with amino acid residue Asp25' of the chain B at a distance of $1.719 \AA$ and $2.469 \AA$ (Fig. 1b), respectively. The binding orientation of lopinavir shows that the carbonyl group (attached to 2-(2,6-dimethylphenoxy)acetamido moiety) forms a good hydrogen bond interaction with the amino acid residue Ile50 at distance of $2.543 \AA$ (Fig. 1c). The free hydroxyl group also forms two hydrogen bonds with a crucial amino acid residue Asp25 of chain A at a distance of $2.287 \AA$ and 1.963 $\AA$, respectively. The carbonyl group of tetrahydro-pyrimidin2(1H)-one moiety also formed a significant hydrogen bond with Gly48 (2.335 $\AA$ ). In case of atazanavir, the amino group of one of the methyl carbamate moiety forms hydrogen bond with the amino acid residue Asp30 of chain A. The methoxy group of the other methyl carbamate moiety also forms an important hydrogen bond with the amino acid residue Ala28' (2.208 ̊) of chain B (Fig. 1d). The indinavir showed interaction with both the chain A and B. The hydroxyl group and the amino group (attached to 2,3-dihydro- $1 H$-inden-2-ol moiety) showed significant hydrogen bond interactions with amino acid residues Gly48 (2.196 ̊), Asp29 (2.474 ̊) and Asp30 (1.839 $\AA$ ), respectively of chain A. The amino group [attached to $N$-(tert-butyl)acetamide] also formed a good hydrogen bond interaction with the amino acid residue Gly27' of chain B at a distance of $2.282 \AA$ (Fig. 1e). The nelfinavir has shown only two hydrogen bonding interactions (Fig. 1f) between phenolic $\mathrm{OH}$ group and $\mathrm{NH}$ of $\mathrm{N}$-(tert-butyl)acetamido group with Gly49 (1.951 A) of chain A and Gly27' $(2.121 \AA)$ of chain $\mathrm{B}$, respectively. It could not show interactions with other crucial amino acid residues which might be the reason of its low activity as compared to other drugs (Table1). Interestingly, ritonavir has shown four significant hydrogen bonding interactions with all the crucial amino acid residues of chain A: Gly49 (2.305 ̊), Ala28 (2.482 ̊), Asp29 (2.185 $\AA$ ) and Asp25 (1.782 ̊), respectively (Fig. 1g). Despite of all these interaction, this compound has low potency $\left(\mathrm{IC}_{50}=4.6\right.$ \pm 1.4 ) and this might be because of the lack of interaction with crucial amino acid residues of chain $B$ as a result of which it is mostly used in combination with lopinavir ${ }^{13}$. The binding orientation of saquinavir shows that it anchored well at the interface of the homodimer (chain A and chain B) but formed all the hydrogen bond interaction with the amino acid residues of chain A only (Fig. 1h). It formed strong hydrogen bonding interactions with amino acid residues Gly48 (2.085 ̊), Asp25 (1.979), Ala28 (2.089 ̊), Asp29 (1.932 ̊) and Asp30 (2.256 $\AA)$, respectively. Surprisingly, the tiprinavir showed only one significant hydrogen bond interaction between the carbonyl group of 6-hydroxy-pyranone moiety and Gly49 at a distance of $2.010 \AA$ (Fig. 1i) even though it anchored well at the interface of chain A and chain B. Even with only one important hydrogen bond interaction, its activity is good which suggests that Gly49 residue of the amino acid A is a crucial for activity which is found to be the key point of interaction in almost all the inhibitors. Table- 2 represents the electrostatic interaction (Coulomb) energy (in $\mathrm{kcal} / \mathrm{mol}$ ) and vdW interaction energy (in $\mathrm{kcal} / \mathrm{mol}$ ) between all the study compounds and each single amino acid involved in ligand recognition obtained after docking simulations inside the binding site of HIV-1 protease. QikProp (version 3.6) module of Schrodinger was used to evaluate certain molecular properties which could influence the metabolism, cell permeability and bioavailability for the FDA approved compounds (Table-3). Some parameters such as $\mathrm{QP} \log \mathrm{Po} / \mathrm{w}$ and $\mathrm{QP} \log \mathrm{S}$ are recognized parameters for prediction of drug transport properties. Further, steric and molecular surface descriptors, e.g., solvent accessible area (SASA) were also calculated. To assess the bioavailability of all the compounds, various ADME properties were also calculated (Table-3). Further, we calculated the compliance of all compounds to Lipinski's rule of five ${ }^{21}$ which has been widely used as a filter for predicting the drug like properties of any molecule. As stated by this rule, poor absorption or permeation is more likely to occur when there are more than five hydrogen bond donors, ten hydrogen bond acceptors, the molecular weight is greater than 500 and the calculated $\log \mathrm{P}$ $(\mathrm{C} \log \mathrm{P})$ is greater than 5. Poor or problems with bioavailability are shown by the molecules violating more than one of these rules. Interestingly, the results of the predicted properties of all FDA approved protease inhibitors are in range as predicted by QikProp for $95 \%$ of the known oral drugs.

TABLE-2

ELECTROSTATIC INTERACTION ENERGY (KCAL/MOL) AND VDW INTERACTIONS BETWEEN THE COMPOUNDS AND CRUCIAL AMINO ACID RESIDUES OF HIV-1 PROTEASE INVOLVED IN LIGAND RECOGNITION

\begin{tabular}{|c|c|c|c|c|c|c|c|c|c|}
\hline \multirow{2}{*}{ Compounds } & \multirow{2}{*}{${ }^{\mathrm{a}} \mathrm{G} \_$score } & \multicolumn{2}{|c|}{ GLY 27} & \multicolumn{2}{|c|}{ ASP 29} & \multicolumn{2}{|c|}{ ASP 30} & \multicolumn{2}{|c|}{ ASP 25, } \\
\hline & & $\mathrm{VdW}^{\mathrm{b}}$ & Coulomb & VdW & Coulomb & VdW & Coulomb & $\mathrm{VdW}$ & Coulomb \\
\hline Amprenavir & -8.1 & -2.341 & -0.409 & -2.123 & -0.060 & -2.095 & -0.630 & -0.724 & -14.593 \\
\hline Darunavir & -8.1 & -2.697 & -0.134 & -3.100 & -4.918 & -1.565 & -4.127 & -2.662 & -1.927 \\
\hline lopinavir & -7.1 & -2.240 & 0.182 & -2.822 & -0.026 & -2.998 & -1.625 & -1.639 & -11.798 \\
\hline Atazanavir & -5.6 & -2.006 & -0.069 & -4.693 & -4.493 & -2.413 & -10.120 & -1.583 & -3.029 \\
\hline Indinavir & -7.4 & -1.341 & -0.351 & -2.201 & -0.469 & -1.268 & -4.186 & -1.850 & -1.123 \\
\hline Nelfinavir & -8.7 & -2.820 & 1.249 & -2.813 & -16.749 & -2.722 & -15.247 & -2.110 & -35.689 \\
\hline Ritonavir & -7.6 & -3.084 & -0.733 & -3.776 & -3.370 & -2.037 & 2.774 & 0.334 & -13.516 \\
\hline Saquinavir & -8.2 & -2.707 & 3.423 & -3.934 & -9.581 & -2.615 & -16.585 & -0.812 & -43.603 \\
\hline Tipranavir & -8.6 & -1.624 & 0.574 & -0.883 & 2.853 & -0.248 & 0.754 & -1.921 & -2.632 \\
\hline
\end{tabular}

${ }^{\mathrm{a}}$ GLIDE XP_Score; ${ }^{\mathrm{b}}$ van der Waals interaction 
TABLE-3

CALCULATION OF VARIOUS ADME PROPERTIES OF FDA THE COMPOUNDS PROTEASE INHIBITORS

\begin{tabular}{|c|c|c|c|c|c|c|c|c|c|c|c|}
\hline Molecule & $\mathrm{MW}^{\mathrm{a}}$ & SASA $^{b}$ & $\begin{array}{c}\text { Donor } \\
\mathrm{HB}^{\mathrm{c}} \\
\end{array}$ & $\begin{array}{c}\text { Accpt } \\
\mathrm{HB}^{\mathrm{d}}\end{array}$ & $\begin{array}{l}\text { QPlogP } \\
\mathrm{o} / \mathrm{w}^{\mathrm{e}}\end{array}$ & $\mathrm{QP} \log \mathrm{S}^{\mathrm{f}}$ & $\mathrm{QPPCaco}^{\mathrm{g}}$ & $\mathrm{QP} \log \mathrm{BB}^{\mathrm{h}}$ & \#metab ${ }^{\mathrm{i}}$ & $\begin{array}{c}\text { Human oral } \\
\text { absorption }(\%)^{\mathrm{j}}\end{array}$ & $\begin{array}{c}\text { Rule of } \\
\text { Five }^{k}\end{array}$ \\
\hline Amprenavir & 505.628 & 717.015 & 3.5 & 11.4 & 2.808 & -3.287 & 262.096 & -1.665 & 4 & 2 & 2 \\
\hline Darunavir & 547.665 & 683.329 & 3.5 & 13.1 & 2.434 & -2.37 & 602.619 & -1.238 & 3 & 2 & 2 \\
\hline lopinavir & 628.81 & 862.674 & 4 & 9.45 & 5.1 & -5.092 & 1138.89 & -1.209 & 8 & 1 & 2 \\
\hline Atazanavir & 718.892 & 1188.07 & 3.5 & 12.7 & 6.547 & -9.052 & 144.019 & -2.87 & 7 & 1 & 2 \\
\hline Indinavir & 615.814 & 1032.85 & 4 & 13.9 & 2.659 & -3.787 & 13.036 & -1.291 & 10 & 1 & 2 \\
\hline Nelfinavir & 567.785 & 813.164 & 4 & 9.95 & 4.012 & -4.096 & 306.217 & -0.257 & 5 & 2 & 3 \\
\hline Ritonavir & 706.917 & 1155.15 & 4.25 & 10.95 & 6.16 & -8.447 & 82.874 & -2.774 & 9 & 1 & 1 \\
\hline Saquinavir & 670.85 & 1047.99 & 5 & 13.7 & 2.503 & -4.006 & 7.492 & -2.346 & 7 & 2 & 2 \\
\hline Tipranavir & 602.667 & 924.78 & 1 & 10.5 & 5.56 & -7.707 & 317.973 & -1.63 & 7 & 1 & 1 \\
\hline
\end{tabular}

${ }^{a}$ Molecular weight; ${ }^{b}$ Total solvent accessible surface area (SASA) in square angstroms using a probe with a $1.4 \AA$ radius, range $95 \%$ of drugs (300.0$1000.0)$; ${ }^{\circ}$ Estimated number of hydrogen bonds that would be donated by the solute to water molecules in an aqueous solution, range $95 \%$ of drugs (0.0-6.0); ${ }^{~}$ Estimated number of hydrogen bonds that would be accepted by the solute from water molecules in an aqueous solution, range $95 \%$ of

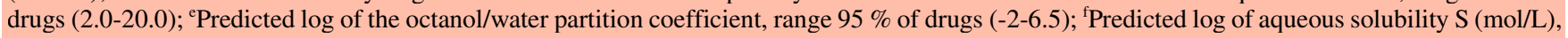
range $95 \%$ of drugs $(-6.5-0.5)$; ${ }^{\circ}$ Caco- 2 cell permeability in $\mathrm{nm} / \mathrm{s}$, range $95 \%$ of drugs $(<25$ poor, $>500$ great). Caco- 2 cells are a model for the gut-

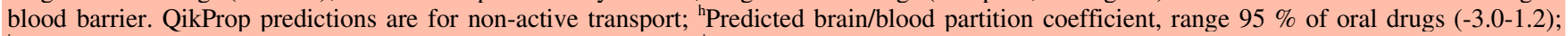

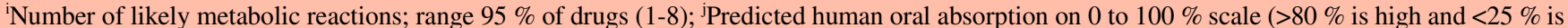
poor); ${ }^{k}$ Number of violations of Lipinski's rule of five. The rules are: mol_MW $<500$, Qplog Po/w $<5$, donor HB $\leq 5$, accpt $\mathrm{HB} \leq 10$. Compounds that satisfy these rules are considered druglike. (The "five" refers to the limits, which are multiples of 5).
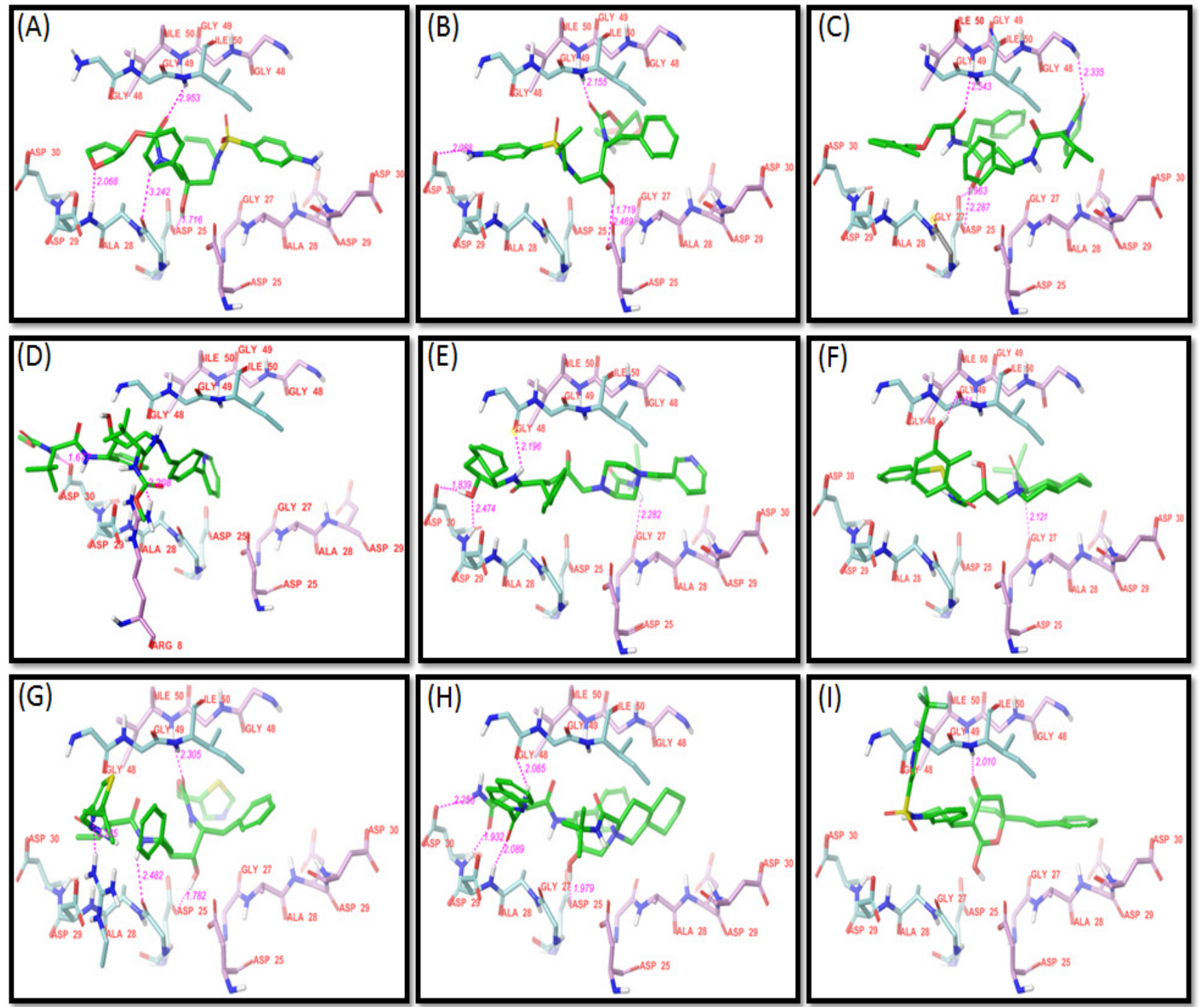

Fig. 1. Binding orientation of all the docked molecules within the binding site of HIV-1 protease (PDB ID: 3 EKV) showing hydrogen bonding interaction with crucial amino acid residues; (a) Ampinavir (b) Darunavir (c) Lopinavir (d) Atazanavir (e) Indinavir (f) Nelfinavir (g) Ritonavir (h) Saquinavir (i)Tiprinavir 


\section{Conclusion}

In the present study, a total of nine (9) FDA approved protease inhibitors and the crystal structure of HIV-1 protease (PDB ID: 3 EKV) was used to carry out in silico molecular docking study by employing the Glide module of Schrodinger software. Prime MM-GBSA approach was used to study the association as well as the free energy of binding of the ligands with the enzyme protease. Binding mode analysis shows that though the active site is located at the interface of the two chains A and B protease; H-binding to both chains is not mandatory for exhibiting the higher potency by inhibiting the enzyme protease. It is evident from the drug nelfinavir which shows hydrogen bond interaction with both the chains A (Gly49) and chain B (Gly27') but the activity is lowest among all the FDA approved protease inhibitors. Similarly, amprenavir though anchored well at the interface but forms all the hydrogen bond interaction with chain A only and shows highest activity. It can be observed that the interactions with the amino acid residues Asp25, Gly27, Ala28, Asp29 and Gly49, respectively is crucial for inhibiting the enzyme proteaseas evident by most of the highly potent inhibitors/drugs (Fig. 1a,b,d and h). Furthermore, to get better sight into physio-chemical requirements for effective binding of all these inhibitors with HIV-1 protease and also to evaluate their drug like acceptability, ADME properties were calculated which was found to be in ranges predicted by QikProp for $95 \%$ of the known oral drugs. Results of this comparative binding mode analysis of all the FDA approved drugs could be potential and useful for the design of new potent inhibitors of HIV-1 protease.

\section{ACKNOWLEDGEMENTS}

One of the authors, P. K. D. is thankful to Pharmaceutical Chemistry Division, School of Pharmacy, IMU, for providing the facilities to carry out the docking studies. This work is an output of CAL-Labs, Drug Design, Discovery and Development, Semester-2, M.Sc. (2013). The authors are also thankful to Mr. Raghurangaswamy, Executive Director, Schrodinger, India for providing the evaluation license (14 Jun-16 july, 2013) to carry out the molecular modelling studies.

\section{REFERENCES}

1. J. Rebehmed, F. Barbault, C. Teixeira and F. Maurel, J. Comput. Aided Mol. Des., 22, 831 (2008).

2. R.A. Weiss, Science, 260, 1273 (1993).

3. Z. Ul-Haq, S. Usmani, H. Shamshad, U. Mahmood and S.A. Halim, Chem. Cent. J., 7, 88 (2013).

4. Y. Mehellou and E. De Clercq, J. Med. Chem., 53, 521 (2010).

5. N.E. Kohl, E.A. Emini, W.A. Schleif, L.J. Davis, J.C. Heimbach, R.A. Dixon, E.M. Scolnick and I.S. Sigal, Proc. Natl. Acad. Sci. USA, 85, 4686 (1988).

6. J.C. Adkins and D. Faulds, Drugs, 55, 837 (1998).

7. J.M. Molina and A. Hill, Expert Opin. Harmacother., 8, 1951 (2007).

8. G.L. Plosker and S. Noble, Drugs, 58, 1165 (1999).

9. P. Tebas and W.G. Powderly, Expert Opin. Pharmacother., 1, 1429 (2000).

10. A.P. Lea and D. Faulds, Drugs, 52, 541 (1996).

11. J.J. Orrick and C.R. Steinhart, Ann. Pharmacother., 38, 1664 (2004)

12. Z. Temesgen, F. Cainelli and S. Vento, Drugs Today, 41, 711 (2005).

13. E.M. Mangum and K.K. Graham, Pharmacotherapy, 21, 1352 (2001).

14. K. Bragman, Adv. Exp. Med. Biol., 394, 305 (1996).

15. S. Becker and L. Thornton, Expert Opin. Pharmacother., 5, 1995 (2004).

16. Maestro Version 9.4 and Glide v5.9, Schrödinger, LLC, New York, NY, (2013).

17. D.P. Kishore, M. Raghuprasad, A.R. Rao and P.M. Rao, Asian J. Chem., 25, 10583 (2013).

18. P.K. Deb, A. Sharma, P. Piplani and R.R. Akkinepally, Mol. Divers., 16, 803 (2012).

19. Prime, Version 3.2 Schrodinger, LLC, New York (2013).

20. QikProp, Version 3.6, Schrödinger, LLC, New York (2013).

21. C.A. Lipinski, F. Lombardo, B.W. Dominy and P.J. Feeney, Adv. Drug Deliv. Rev., 46, 3 (2001). 